\title{
Are generic topical prostanoids the way forward in the care of glaucoma patients? - No
}

Eye (2013) 27, 1002-1003; doi:10.1038/eye.2013.129; published online 28 June 2013

Given below are examples of clinical scenarios that I have encountered since starting my position as a consultant in March 2012. These scenarios aim to show that the use of cheaper generic preparations may not automatically lead to a cost-saving owing to a requirement for more outpatient appointments. Latanoprost is being cited as an example because of its widespread use.

Scenario 1. A patient stops using the generic latanoprost, as the bottle looks different from their usual Xalatan, but does not tell anyone until they come for their follow-up appointment.

Consequence: the patient's condition has been untreated for some weeks/months with an unquantifiable effect on disease progression. The outpatient appointment is wasted, as without applanation tonometry representing a treatment state the clinician cannot make a clinical decision as to whether the treatment is effective. There are cost implications of a clinic appointment to consider (which is charged to the commissioner, who also pays for the latanoprost) along with a wasted clinic slot that may have been better utilised.

Scenario 2. A patient is unable to use the generic latanoprost because of not being to hold/squeeze the bottle properly, owing to the shape of the bottle being different from usual.

Consequence: as above.

Scenario 3. A patient is unable to use generic latanoprost, as the bottle does not fit the compliance aid issued with the original Xalatan.

Consequence: as above.

Scenario 4. A patient uses too much generic latanoprost because of the bottle releasing too much medication, and the medication runs out before the repeat prescription is due.

Consequence: extra costs are incurred owing to (previously unnecessary) interim prescriptions. One patient was unable to obtain extra eye drops, as their family doctor refused to provide an interim prescription.

Scenario 5. A patient receives a different generic product from 1 month to the next, thereby compounding scenarios 1-4 with the same results. Patients become confused with and wary of their family doctors/pharmacists as a result of changes in packaging.

Scenario 6. Generic preparations do not all have the same storage instructions. Patients who are used to storing their Xalatan at room temperature-once opened-may be incorrectly assuming that this applies to all versions of generic latanoprost.

Consequence: the patient may not have been treating their glaucoma with as much active agent as usual.

Scenario 7. A previously well-controlled patient on Xalatan is now switched onto generic latanoprost and is no longer controlled despite good reported compliance, adequate supply, and a suitable storage environment. Possible reasons for this loss of control include a natural decline in the patient's condition, or the generic latanoprost is not as effective as Xalatan. It is impossible to tell the cause of the patient's loss of control, although I have experienced an improvement in patients' control following a switch to a branded alternative. Whether this is due to a compliance issue or a more efficacious product is impossible to ascertain. An extra outpatient appointment has to happen to make this otherwise unnecessary change, wasting resources.

Scenario 8. Dispensing errors: I have now seen two patients who were both prescribed latanoprost on a repeat basis by their family physician; both were using fixed-combination generic latanoprost and timolol and both were experiencing side effects from the use of betablockers; both of these cases were due to dispensing errors made by different pharmacists in different pharmacies. Both drug boxes were clearly labelled with latanoprost only. See Figure 1. 


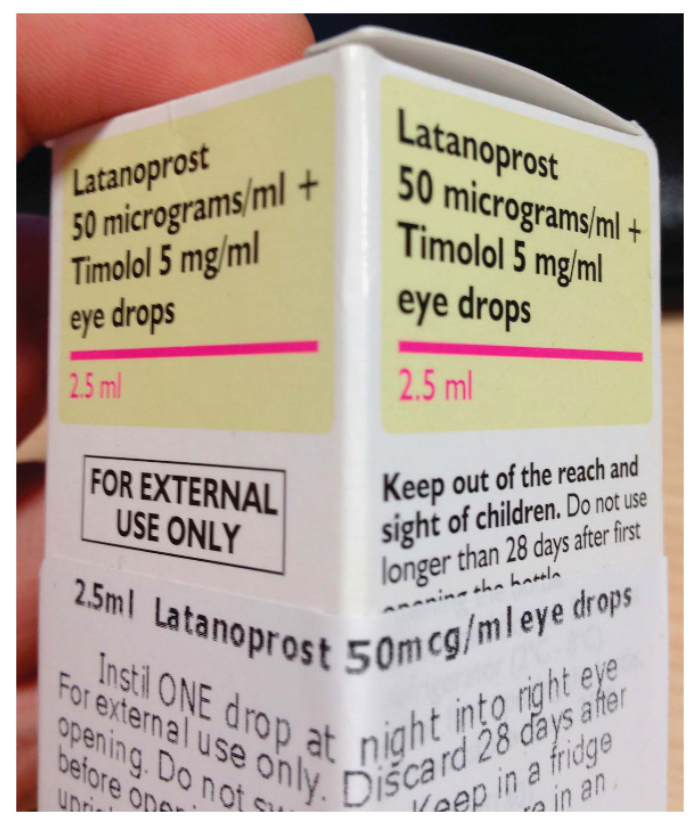

Figure 1 An example of a dispensing error.

\section{Discussion}

With 20 product licences for latanoprost granted by the MHRA, ${ }^{1}$ pharmacists now have multiple latanoprost options to choose from. Because of contracts with their suppliers, pharmacists may have little control over which generic product they can stock. In addition, the brand of product stocked may change from one month to the next according to market forces. Should patients have to try several pharmacies to obtain their regular generic product?

It stands to reason that if there is both a frequent change in the appearance of latanoprost packaging, combined with very little difference in the packaging of the monotherapy and the fixed-combination agent of the same brand, then dispensing errors can creep in.

My concerns about generic latanoprost are not related to the safety of the active agent, nor do they concentrate on the efficacy of generic latanoprost. However, an efficacious drug needs to be consistently usable by patients.

These problems can be overcome. The point is that compliance is a difficult issue in glaucoma care. Medical and allied health professionals, the International Glaucoma Association, and the pharmaceutical industry devote significant time, effort, and hence money to compliance. The last thing a glaucomatologist needs is a set of unknown variables to exacerbate this problem.

I appreciate the cost-saving reasons for using generic preparations, but I feel that the cost-saving is rapidly annulled through wasted appointment slots. At a time where capacity generation within ophthalmology outpatient departments is a priority for many units in the United Kingdom, avoiding unnecessary outpatient appointments is crucial.
In some countries, such as Australia, prescribers and patients can allow for generic substitution of a branded item. As we do not have that facility when prescribing in the United Kingdom, I exercise that choice by avoiding a product with a known generic alternative. I appreciate that it is not possible to prevent the manufacture of generic products. I am not against generic products per se, only the issues raised within this article resulting from the use of generic latanoprost.

Has generic prescribing been relevant to other topical antihypertensives? Timolol was launched in 1979 and came off patent in 1996. Pfizer started marketing Xalatan in 1996. If there were any such compliance problems at the time, it is possible that these were eclipsed by the huge impact that Xalatan had on glaucoma care.

Compliance issues in the context of generic tablet medications may be prevalent, but as an ophthalmologist I am largely unaffected by this. I venture that owing to one's innate ability to feed oneself it requires less skill to swallow a tablet than to instil an eye drop. Hence, it is not unreasonable to think that compliance in ophthalmology is more challenging compared with disciplines where tablet-administered treatments are the norm.

I did not foresee any of the above concerns, nor did I think that the availability of generic latanoprost would result in a decline in my use of latanoprost, yet this is what has happened. Owing to concerns raised over patient safety at Aintree University Hospitals NHS Foundation Trust on grounds of poor compliance, we no longer prescribe latanoprost.

\section{Conflict of interest}

The author does not have any proprietary interests in any of the products mentioned. He has obtained reimbursement for attending symposia from Pfizer, MSD, Allergan, and Alcon.

\section{Reference}

1 Advice on generic latanoprost prescribing. The Royal College of Ophthalmologists Professional Standards Committee \& Lucy Titcomb, U.K. Ophthalmic Pharmacy Group of the Royal Pharmaceutical Society. April 2012, Available at http:/ / www.rcophth.ac.uk/page.asp?section $=442 \&$ sectionTitle $=$ Patient+Safety+Information.

VDJ-P Dubois

Aintree University Hospital NHS Foundation Trust, Longmore Lane, Liverpool, Merseyside, UK

Correspondence: VDJ-P Dubois, Consultant Ophthalmologist and Glaucoma Lead, Aintree University Hospital NHS Foundation Trust, Longmore Lane, Liverpool, Merseyside L9 7AL, UK.

$$
\text { Tel: + } 44 \text { (0)151 } 529 \text { 0147; }
$$

Fax: + 44 (0)151 5290139 .

E-mail: vincent.dubois@aintree.nhs.uk 